\title{
REVIEW
}

\section{Downstream EWS/FLI1 - upstream Ewing's sarcoma}

Heinrich Kovar*

\begin{abstract}
Ewing's sarcoma family tumors are a good example of how genome research has advanced our understanding of the molecular pathogenesis of an otherwise enigmatic disease. This group of embryonal bone tumors is characterized by the expression of a chimeric ETS-family oncogene, predominantly EWS/FL I1. There is now convincing evidence for a mesenchymal descent from an early pluripotent progenitor. EWS/FLI1 has been shown to drive proliferation of Ewing's sarcoma cells and block most of the differentiation potential except for a partial neural gene expression program. The EWS/FLI1 fusion protein acts mainly as a gene activator, directly interacting with chromatin at two kinds of binding site: distant enhancers enriched in GGAA microsatellites, and proximal promoters containing classical ETS-binding motifs and recognition motifs for other transcription factors. EWS/FLI1 also represses a large number of genes, mainly indirectly, presumably by altering microRNA expression and epigenetic mechanisms, and potentially affecting post-transcriptional gene regulation. Modulation of EWS/FLI1 expression is not only a desirable therapeutic goal, but may also occur under physiological conditions and influence the course of the disease.
\end{abstract}

\section{Introduction}

Ewing's sarcoma family tumors (ESFTs) are a group of mostly undifferentiated, highly aggressive, small roundcell tumors affecting mostly children and adolescents, with a peak incidence around 15 years of age [1]. Their origin has been a matter of debate ever since they were first described in 1921 by James Ewing [2]. Although presenting predominantly as a neoplasm of the bone, the exact tumor histogenesis remains poorly defined, and rare occurrences in the soft tissue of other organs [3] point to a pluripotent migratory cell of origin.

*Correspondence: heinrich.kovar@ccri.at

Children's Cancer Research Institute, St Anna Kinderkrebsforschung, Zimmermannplatz 10, 1090 Vienna, Austria
The unifying genetic trait of this family of tumors is a chromosomal translocation, $\mathrm{t}(11 ; 22)(\mathrm{q} 24 ; \mathrm{q} 12)$, that was first described more than 20 years ago [4] and molecularly elucidated in 1992 [5]. The genomic rearrangement results in the fusion of two genes, EWS and FLI1. EWS encodes a RNA-binding protein that associates with components of the basal transcriptional machinery [6-8] and post-transcriptional RNA processing [9-12], and $E W S$ knockout mice are deficient in homologous recombination and recombination repair [13]. FLI1 encodes a member of the ETS protein family, a group of winged helix-loop-helix transcription factors sharing a DNAbinding domain with specificity for GGAA/T core motifs. In the ESFT-specific EWS/FLI1 fusion protein, the EWS RNA-binding domain is replaced by the FLI1 DNAbinding domain, thus creating a novel ETS transcription factor with unique properties. In about 10-15\% of ESFTs, one of four related ETS transcription factors (ERG, ETV1, ETV4 or FEV) substitutes for FLI1 in alternative but identically structured EWS fusion proteins [14].

\section{EWS/FLI1 shapes the ESFT phenotype}

Functional studies of ectopically expressed EWS/FLI1 using promiscuous ETS binding sites to drive reporter gene activity revealed that the amino-terminal EWS domain contributes strong transcriptional activation properties to EWS/ETS fusion proteins [15-17]. Early expression profiling studies of EWS/FLI1-transduced cell line models confirmed that a plethora of genes are upregulated by the fusion protein. However, an almost equal number of genes were consistently found to be repressed [18-21]. These approaches did not discriminate between direct and indirect activities of the chimeric oncogene and thus the EWS/FLI1-dependent mechanisms underlying aberrant gene expression in these model systems remained elusive. They also did not account for the fact that tolerance to EWS/FLI1 expression and the pattern of responsive genes depends on the cellular context [22].

A common observation in these studies was that ectopic EWS/FLI1 imposed neuronal and endothelial features of gene expression on non-ESFT cells $[19,22]$. These results may provide a molecular explanation for James Ewing's original phenotypic classification of the entity as "endothelioma of the bone" [2], which was later 
supported by ultrastructural findings in the 1970s and 1980s. It also provides a mechanistic foundation for the prevailing immunohistochemistry-based view of the 1990s of a neuroectodermal origin of the disease, which was strengthened by anecdotal reports about chemotherapy-or experimentally-induced neural differentiation of ESFT cells.

\section{Mesenchymal origin of ESFT and the EWS/FLI1 signature}

The profiling data obtained from models that ectopically express EWS/FLI1 have suggested that partial endothelial and neural differentiation are a consequence of the transcriptional activity of the fusion oncogene, largely independent of the histological background. This hypothesis obtained support when it became possible to modulate endogenous EWS/FLI1 expression in ESFT cells by means of RNA interference.

Among studies that used this approach, two investigations also considered gene expression of primary ESFTs in comparison with a series of normal human tissues and arrived at a similar conclusion: the tissue whose expression is most similar to that shown by ESFTs when EWS/ FLI1 is turned off is mesenchymal progenitor cells (MPCs) [23,24]. Tirode et al. [24] demonstrated that although ESFT cells are blocked in their differentiation potential towards adipogenic and osteogenic lineages, they regain pluripotency upon long-term inhibition of EWS/FLI1 expression. This finding is consistent with earlier experimental results that demonstrated the blockage of mesenchymal differentiation potential of EWS/ FLI1-transformed MPCs [25] and the acquisition of ESFT-like gene expression and tumorigenic properties of these cells in mice [26,27]. Kauer and colleagues [23] demonstrated that, when MPCs are chosen as a reference tissue, most differences in gene expression of ESFTs are caused by the expression of EWS/FLI1, with few exceptions. On the basis of this comparison, an ESFT-specific EWS/FLI1 gene expression signature was obtained. At a significance level of $P=0.1,344$ and 237 genes were found to be up- and down-regulated by the fusion oncogene, respectively. Interestingly, only a third of EWS/ FLI1-induced genes and a fifth of the repressed genes from this single-platform analysis overlapped with a signature previously published by Hancock et al. [28], which was based on a meta-analysis of EWS/FLI1dependent gene expression in various models and analyzed on various expression-profiling platforms. Although there was a strong overall overlap in the gene lists affected by EWS/FLI1, about $80 \%$ of the EWS/FLI1 signature genes in the earlier study [28] did not achieve the significance threshold of the Kauer analysis [23], and $70 \%$ of the Kauer signature [23] was not found to be significant in the Hancock dataset [28]. This discrepancy is probably due to the use of different reference tissues, given that the Hancock study [28] compared ESFTspecific gene expression with the mean of many tissues. This corroborates the importance of considering the influence of the cellular background in the interpretation of EWS/FLI1 target gene studies. It also highlights the necessity of choosing an adequate reference tissue for target validation in primary ESFT.

With an incidence of 1.3 per million population, ESFTs are rare tumors [29]. Diagnostic samples are most frequently obtained by fine needle biopsy and, therefore, adequate materials for genomic studies are often difficult to obtain. However, the establishment of a reliable gene expression signature for ESFT and the validation of robustly EWS/FLI1-regulated genes require the analysis of large cohorts of primary tumors from untreated patients on a single analysis platform. Such series are not common within a single institution. So far there are only four expression datasets from comparable screening platforms (Affymetrix HGU-133-A and HGU-133-PLUS2) publicly available, together comprising almost 100 tumors [24,30-32]. These data are a valuable resource for target validation studies. However, only two of these datasets are linked to clinical parameters [31,32]; one has been used to define a prognostic signature suggesting a role for the glutathione metabolic pathway in chemotherapy resistance [32]. This and similar future studies will require independent confirmation on clinically wellannotated tumors from large, uniformly treated patient cohorts. Thus, the field of ESFT research is likely to profit tremendously from the ongoing multicentric European and Children's Oncology Group clinical trials being successful in generating and depositing gene expression data linked to stage and outcome information on their patient cohorts in publicly accessible data banks.

\section{A role for EWS/FLI1 in post-transcriptional gene regulation?}

Ideally, ESFT gene expression data banks should also contain raw data from analyses on exon arrays to account for variations in alternative splicing and/or promoter usage. Previous experimental studies have demonstrated that EWS/FLI1 can affect 5' splice site selection in vitro [33], and the first example of EWS/FLI1-dependent transcription-coupled alternative splicing in ESFT was described for its direct target gene cyclin D1 [9,34]. This finding was not unexpected given the multiple interactions of the fusion oncogene with components of the splicing machinery inherited from the parental EWS protein [35-37]. Given that the FLI1 DNA-binding domain recruits these factors to its chromatin targets through interaction with the EWS amino-terminal domain, it is likely that the number of alternatively spliced tumorspecific EWS/FLI1 targets identified will increase as soon 
as comprehensive exon expression data become available. Even more information about the abundance, identity and structure of ESFT-specific mRNA and non-coding RNA species will be gained upon application of nextgeneration sequencing technology to the analysis of the ESFT transcriptome. Exon-based analysis will shed light on the poorly understood link between transcriptional and post-transcriptional control of gene regulation by EWS/FLI1.

\section{Lessons from the analysis of EWS/FLI1 chromatin interactions}

So far, the use of next-generation sequencing for the analysis of EWS/FLI1 binding regions in the ESFT chromatin has yielded surprising results: whereas previous chromatin immunoprecipitation (ChIP) studies followed by gene-specific PCR (ChIP-PCR) identified EWS/FLI1 binding in proximal promoter and intronic regions of specific targets, including $T N C$ [38], Id2, cMYC, CCND1 and TGFBR2 [39], CDKN1A [40], IGFBP3 [41], PTPL1 [42], STYXL1 [43], AURKA and AURKB [44], GLI1 [45] and EZH2 [46], the first published nextgeneration sequencing study of genomic DNA recovered by ChIP (ChIP-seq) using a FLI1-specific antibody detected EWS/FLI1 binding sites in ESFT chromatin predominantly far away from the transcriptional start sites of genes, with a mean distance of $242 \mathrm{~kb}$ and up to $3 \mathrm{Mb}$ [47]. More than half of the EWS/FLI1 binding sites were localized to intergenic regions. Strikingly, 104 of 246 identified binding regions consisted of microsatellites comprising three or more GGAA repeats, the core ETS binding motif [47]. This result was consistent with previous ChIP-chip (hybridization of ChIP products to DNA microarrays) results obtained with the same antibody, which also identified GGAA microsatellites as highly over-represented among EWS/FLI1 binding regions [48].

The authors of the two studies $[47,48]$ attempted to functionally validate the potential transcription enhancing activity of GGAA microsatellites in response to EWS/ FLI1 by testing multimers of the GGAA core motif in luciferase reporter assays [49]. The discovery that GGAA microsatellites are associated with EWS/FLI1 protein in ESFT chromatin and have the potential to drive gene transcription when directly fused to a minimal promoter in in vitro assays was a new and exciting addition to a growing body of evidence supporting the idea that sequences previously considered as genomic 'junk' might indeed have important functions [50]. The question remains whether binding to these elements in the chromatin context is sufficient to activate gene transcription from a distance of several hundred kilobases, and whether this is the predominant mode of aberrant gene regulation by the oncogene in ESFT. Functional proof that the observed EWS/FLI1 binding to such microsatellites affects gene regulation in vivo requires testing of endogenous gene activity following targeted deletion of the respective microsatellite from the genomic region in living cells. Furthermore, the identification of genes regulated by this putative 'enhancer-type' mechanism is complicated by the fact that a majority of EWS/FLI1 binding GGAA microsatellites localize to intergenic regions and cannot be assigned to specific genes. Therefore, the functional impact of this observation remains to be defined.

When comparing gene expression signatures of EWS/ FLI1 with the published ChIP-chip and ChIP-seq results, less than $10 \%$ of EWS/FLI1-regulated genes were found to be binding targets by these methods [23], suggesting that either the vast majority of EWS/FLI1-responsive genes are indirectly regulated or a large number of direct EWS/FLI1 targets were missed. From a mechanistic point of view, repetitive DNA sequences with high GGAA content may be high affinity binding sites for the chimeric ETS transcription factor and, therefore, ChIP-based identification procedures may selectively enrich for these genomic regions. In fact, an unpublished ChIP-seq analysis (Bilke S, Kauer M, Kovar H, Meltzer P, personal communication), which increases the number of sequencing reads for EWS/FLI1 more than 30-fold over the previously published Guillon et al. study [47], identified almost equal amounts of EWS/FLI1 binding sites close to transcriptional start sites of genes and in intragenic regions (over $4 \mathrm{~kb}$ from the TSS). Importantly, all previously identified (microsatellite) binding regions were also found. Given that, in this study (Bilke et al., personal communication), the number of binding regions increased with the number of sequencing runs performed, and several previously identified direct EWS/ FLI1 target promoters were recovered only with increasing runs, it is possible, although unproven, that promoter hits harboring only few ETS binding motifs be lowaffinity binding sites for the fusion oncogene, whereas GGAA microsatellites are more strongly bound.

\section{Mechanisms of EWS/FLI1-driven gene regulation and functions}

Direct binding of EWS/FLI1 to proximal promoter regions has been inferred from the in silico analysis of upstream regulatory regions of EWS/FLI1 signature genes, which were found to be significantly enriched in bona fide ETS binding motifs [23]. Unpublished kinetic studies of gene expression on inducible EWS/FLI1 knockdown in ESFT cell lines (Kauer M, Schwentner R, Walker RL, Meltzer P, Kovar H, personal communication) have revealed that CGGAAT motifs are particularly enriched in early down-regulated genes, identifying them as candidates for being directly activated by EWS/FLI1. Interestingly, upon EWS/FLI1 knockdown, this group of 
early down-regulated genes showed co-enrichment of binding motifs for the transcription factors E2F, NRF1 and NFY, suggesting transcription factor cooperation in EWS/FLI1-driven gene activation; this remains to be experimentally tested [23]. In addition, the ChIP-seq study by Guillon et al. [47] identified frequent occurrence of combinations of two ETS binding sites in non-microsatellite EWS/FLI1 binding regions, providing the first evidence that the chimeric oncoprotein might activate gene transcription from at least some promoters as a homo- or heterodimer with other ETS transcription factors.

In contrast, EWS/FLI1-repressed genes did not show any enrichment of ETS binding motifs in the in silico analysis [23] or in the ChIP-seq study [47]. Instead, binding motifs for a large number of other transcription factors were identified in the upstream regulatory regions of these genes [23]. These results suggest that the mechanism of EWS/FLI1-mediated gene repression is indirect.

One such mechanism has been identified that involves the EWS/FLI1-activated gene NKX2.2, which encodes a dual-function homeodomain transcription factor [51]. NKX2.2 transcriptional repression functions through recruitment of Groucho-family corepressors. Following knockdown of endogenous NKX2.2 expression in ESFT cell lines, constructs lacking the transactivation domain but retaining the DNA binding and the transcriptional repression domains were able to rescue anchorageindependent growth. About half of 159 NKX2.2-downregulated genes overlapped with EWS/FLI1-repressed genes in ESFT cell lines [51].

Another intriguing mode of EWS/FLI1-induced gene repression may involve epigenetic mechanisms because several genes that encode histone-modifying enzymes are among EWS/FLI1-activated targets. One of them, the histone methyl transferase Enhancer of Zeste homolog 2 (EZH2) gene, has only recently been confirmed [46]. $\mathrm{EZH} 2$, as the enzymatic subunit of the polycomb PRC2 complex, methylates histone H3 Lys27, thereby mediating gene silencing. Silencing of EZH2 in ESFT cells resulted in a generalized loss of methylation on H3 Lys27 as well as an increase in $\mathrm{H} 3$ acetylation, leading to gene activation [52].

Other mechanisms of gene repression mediated by EWS/FLI1 may emerge from the identification of microRNAs (miRNAs) regulated by EWS/FLI1 in ESFTs. Our own preliminary data suggest about 30 miRNAs that are both modulated in response to EWS/FLI1 knockdown in several ESFT cell lines and aberrantly expressed in primary ESFTs when compared with MPCs. However, to fully appreciate the role of these miRNAs in ESFTspecific gene expression, large-scale proteomic data for ESFTs will be required, because most miRNAs are thought to affect protein translation rather than mRNA levels.
The gene sets repressed and activated by EWS/FLI1 differ not only in the mechanisms of regulation by the chimeric oncogene, but also in their biological consequences. Functional annotation of the EWS/FLI1 signature genes revealed that activated genes are predominantly involved in growth-related functions, including proliferation and energy metabolism, consistent with an enrichment of sites for the cell-cycle-promoting transcription factor E2F in EWS/FLI1-induced genes and with the fast growth of ESFTs. In contrast, the functions of EWS/FLI1-repressed genes include developmental and signaling processes [23]. These results are consistent with the highly undifferentiated phenotype of ESFT cells and their inability to differentiate in response to adipogenic and osteogenic stimuli.

Thus, EWS/FLI1 combines in a single molecule two key functions of oncogenic transformation - activated proliferation and differentiation arrest - by different mechanisms. Is this a sufficient condition to trigger malignant transformation and tumorigenicity of the ESFT precursor cell?

\section{Second hit mutations in the development of ESFT}

Riggi et al. [26] introduced EWS/FLI1 as a single oncogene in MPCs from C57BL/6 mice and transplanted them subcutaneously into severe combined immunodeficiency (SCID) mice, which lack an immune system; this resulted in rapid (within 6 weeks) tumor formation. This finding suggests that, in mice, no other mutations may be required for EWS/FLI1-induced tumorigenesis. In contrast, although it has been shown that EWS/FLI1 (and also EWS/ERG) transgenic mice can develop neoplasms without additional impairment of the tumor suppressor p53, latencies were extremely long [53-55]. Similarly, although introduction of EWS/FLI1 into human MPCs resulted in a small round-cell phenotype and a transcriptional profile similar to ESFTs, these cells were not fully transformed, retained their ability to differentiate toward the chondrocytic, osteocytic and adipocytic lineages, and did not form tumors in mice [27]. These results may suggest that, in humans, additional genetic events other than EWS/FLI1 are required to cause tumorigenesis.

Although it has been known for a long time that the number of chromosomal aberrations in ESFT is usually small, a recent cytogenetic study [56] and the only published array comparative genome hybridization study [57] both identified both numerical and structural secondary aberrations in about $80 \%$ of patients. Consistent with earlier studies [58,59], the predominant numerical aberrations were trisomies 8 and 12, which were seen in about half and a third of patients, respectively, as well as gains of chromosomes 2 and 1q. So far, no candidate aberrantly expressed or mutated genes on 
these chromosomes have been identified. However, the first study that used ChIP to identify EWS/FLI1 target genes on a genome-wide scale found an enrichment of EWS/FLI1-activated genes on chromosome 8, suggesting that trisomy may further increase gene dosage of these targets [43]. Structural aberrations consisted mainly of losses at $1 \mathrm{p} 36,9 \mathrm{p} 21,16 \mathrm{q} 2$ and $17 \mathrm{p} 13$, suggesting the involvement of tumor-suppressor genes in these regions. Copy-number alterations at 9p21 and 17 p13 have been associated with loss of the tumor-suppressor gene INK4A and mutations in $p 53$ at frequencies of approximately $25 \%$ and less than 10\%, respectively (for a review, see [60]). Potential tumor suppressors involved in ESFT pathogenesis on chromosomes 1 p36 and $16 \mathrm{q}$ remain to be defined.

\section{Conclusions}

Although the histogenetic origin of ESFTs and the mechanisms of EWS/FLI1-driven oncogenesis have started to crystallize from the genomic studies performed on ESFTs so far, many questions remain, posing a challenge for future therapeutic exploitation.

Although EWS/FLI1 was found to be the major driver of ESFT oncogenesis, genetic and environmental factors influencing EWS/FLI1 levels and activity remain to be identified. Relevant small nucleotide or copy-number polymorphisms may be discovered in genome-wide association studies, and these might ultimately require a community effort to be successful. With respect to environmental factors, a recent unpublished study (Aryee DNT, Niedan S, Kauer M, Schwentner R, Bennani-Baiti IM, Ban J, Muehlbacher K, Kreppel M, Walker RL, Meltzer P, Poremba C, Kofler R, Kovar H, personal communication) demonstrated that ESFTs are frequently hypoxic in patients and that in vitro low oxygen modulates EWS/FLI1 protein levels and the expression of signature genes in ESFT cell lines in a manner dependent on the transcription factor hypoxia-inducible factor 1 (HIF-1 $\alpha$ ). EWS/FLI1 has also been demonstrated to be post translationally modified by phosphorylation [61], and addition of $\mathrm{N}$-acetylglucosamine (GlcNAc) affects the transcriptional activity of the fusion protein [62]. Therefore, pharmacological inhibitors of enzymes responsible for these modifications, such as glutamine analogs that inhibit UDP-GlcNAc biosynthesis, may hold promise as intriguing additions to current polychemotherapy of ESFT [62]. However, the upstream signaling pathways responsible for post-translational modifications of EWS/FLI1 have not yet been defined.

It is intriguing to speculate that microenvironmental factors might influence EWS/FLI1 expression or activity in the tumor tissue directly, via translational (miRNAs) or post-translational mechanisms, or indirectly, via modulation of cooperating transcription factors. Such factors may affect tumor aggressiveness and prognosis and have a role in therapy resistance and relapse. The lack of knowledge about these in vivo factors may be the reason why a drug (cytarabine) that modulates EWSFLI1 protein levels in vitro, as shown by a small compound screen based on EWS/FLI1 signatures, has so far failed to show any significant therapeutic activity against ESFTs in patients $[63,64]$. Thus, further genomic studies, particularly on large cohorts of clinically wellannotated primary tumors, and also post-genomic investigations on signaling pathways in ESFT, are warranted to appreciate the impact of genetic and microenvironmental variations. Ultimately, the emerging knowledge may be translated into a better staging of the disease and the development of new drugs interfering with EWS/FLI1 activity and consequently tumor growth.

\section{Abbreviations}

ChIP, chromatin immunoprecipitation; ChIP-chip, chromatin immunoprecipitation with subsequent hybridization to DNA arrays; ChIP-seq, chromatin immunoprecipitation with subsequent massively parallel sequencing of ChIP products; ESFTs, Ewing's sarcoma family tumors; GICNAC, $\mathrm{N}$-acetylglucosamine; miRNA, microRNA; MPCs, mesenchymal progenitor cells.

\section{Competing interests}

The author declares that he has no competing interests.

\section{Author contribution}

HK drafted the manuscript.

\section{Acknowledgements}

I thank S Bilke, P Meltzer, M Kauer and D Aryee for giving permission to refer to their so-far unpublished results.

Published: 28 January 2010

\section{References}

1. Bernstein M, Kovar H, Paulussen M, Randall RL, Schuck A, Teot LA, Juergens H: Ewing's sarcoma family of tumors: current management. Oncologist 2006, 11:503-519.

2. Ewing J: Diffuse endothelioma of bone. Proc NY Pathol Soc 1921, 21:17-24

3. Kovar H: Ewing's sarcoma and peripheral primitive neuroectodermal tumors after their genetic union. Curr Opin Oncol 1998, 10:334-342.

4. Turc Carel C, Aurias A, Mugneret F, Lizard S, Sidaner I, Volk C, Thiery JP, Olschwang S, Philip I, Berger MP, Philip T, Lenoir GM, Mazabraud A: Chromosomes in Ewing's sarcoma. I. An evaluation of 85 cases of remarkable consistency of $\mathrm{t}(11 ; 22)(\mathrm{q} 24 ; \mathrm{q} 12)$. Cancer Genet Cytogenet 1988, 32:229-238.

5. Delattre O, Zucman J, Plougastel B, Desmaze C, Melot T, Peter M, Kovar H, Joubert I, De Jong P, Rouleau G: Gene fusion with an ETS DNA-binding domain caused by chromosome translocation in human tumours. Nature 1992, 359:162-165.

6. Araya N, Hirota K, Shimamoto Y, Miyagishi M, Yoshida E, Ishida J, Kaneko S, Kaneko M, Nakajima T, Fukamizu A: Cooperative interaction of EWS with CREB-binding protein selectively activates hepatocyte nuclear factor 4-mediated transcription. J Biol Chem 2003, 278:5427-5432.

7. Bertolotti A, Melot T, Acker J, Vigneron M, Delattre O, Tora L: EWS, but not EWS-FLI-1, is associated with both TFIID and RNA polymerase II: interactions between two members of the TET family, EWS and hTAFII68, and subunits of TFIID and RNA polymerase II complexes. Mol Cell Biol 1998, 18:1489-1497.

8. Lee J, Rhee BK, Bae GY, Han YM, Kim J: Stimulation of Oct-4 activity by Ewing's sarcoma protein. Stem Cells 2005, 23:738-751.

9. Sanchez G, Bittencourt D, Laud K, Barbier J, Delattre O, Auboeuf D, Dutertre M: Alteration of cyclin D1 transcript elongation by a mutated transcription factor up-regulates the oncogenic D1b splice isoform in cancer. Proc Natl 
Acad Sci USA 2008, 105:6004-6009

10. Spahn L, Petermann R, Siligan C, Schmid JA, Aryee DN, Kovar H: Interaction of the EWS NH2 terminus with BARD1 links the Ewing's sarcoma gene to a common tumor suppressor pathway. Cancer Res 2002, 62:4583-4587.

11. Pahlich S, Quero L, Roschitzki B, Leemann-Zakaryan RP, Gehring H: Analysis of Ewing sarcoma (EWS)-binding proteins: interaction with hnRNP M, $\mathrm{U}$, and RNA-helicases p68/72 within protein-RNA complexes. J Proteome Res 2009, 8:4455-4465.

12. Erkizan HV, Kong Y, Merchant M, Schlottmann S, Barber-Rotenberg JS, Yuan L, Abaan OD, Chou TH, Dakshanamurthy S, Brown ML, Uren A, Toretsky JA: A small molecule blocking oncogenic protein EWS-FLI1 interaction with RNA helicase A inhibits growth of Ewing's sarcoma. Nat Med 2009, 15:750-756.

13. Li H, Watford W, Li C, Parmelee A, Bryant MA, Deng C, O'Shea J, Lee SB: Ewing sarcoma gene EWS is essential for meiosis and B lymphocyte development. J Clin Invest 2007, 117:1314-1323.

14. Wang L, Bhargava R, Zheng T, Wexler L, Collins MH, Roulston D, Ladanyi M: Undifferentiated small round cell sarcomas with rare EWS gene fusions: identification of a novel EWS-SP3 fusion and of additional cases with the EWS-ETV1 and EWS-FEV fusions. J Mol Diagn 2007, 9:498-509.

15. Bailly RA, Bosselut R, Zucman J, Cormier F, Delattre O, Roussel M, Thomas G, Ghysdael J: DNA-binding and transcriptional activation properties of the EWS- FLI-1 fusion protein resulting from the $t(11 ; 22)$ translocation in Ewing sarcoma. Mol Cell Biol 1994, 14:3230-3241

16. May WA, Gishizky ML, Lessnick SL, Lunsford LB, Lewis BC, Delattre O, Zucman J, Thomas G, Denny CT: Ewing sarcoma 11;22 translocation produces a chimeric transcription factor that requires the DNA-binding domain encoded by FLI1 for transformation. Proc Natl Acad Sci USA 1993, 90:5752-5756

17. Ohno T, Rao VN, Reddy ES: EWS/Fli-1 chimeric protein is a transcriptional activator. Cancer Res 1993, 53:5859-5863.

18. Braun BS, Frieden R, Lessnick SL, May WA, Denny CT: Identification of target genes for the Ewing's sarcoma EWS/FLI fusion protein by representational difference analysis. Mol Cell Biol 1995, 15:4623-4630.

19. Hu-Lieskovan S, Zhang J, Wu L, Shimada H, Schofield DE, Triche TJ: EWS-FLI1 fusion protein up-regulates critical genes in neural crest development and is responsible for the observed phenotype of Ewing's family of tumors. Cancer Res 2005, 65:4633-4644.

20. Lessnick SL, Dacwag CS, Golub TR: The Ewing's sarcoma oncoprotein EWS/FLI induces a p53-dependent growth arrest in primary human fibroblasts. Cancer Cell 2002, 1:393-401.

21. Rorie CJ, Thomas VD, Chen P, Pierce HH, O'Bryan JP, Weissman BE: The Ews/Fli-1 fusion gene switches the differentiation program of neuroblastomas to ewing sarcoma/peripheral primitive neuroectodermal tumors. Cancer Res 2004, 64:1266-1277.

22. Teitell MA, Thompson AD, Sorensen PH, Shimada H, Triche TJ, Denny CT: EWS/ETS fusion genes induce epithelial and neuroectodermal differentiation in NIH 3T3 fibroblasts. Lab Invest 1999, 79:1535-1543.

23. Kauer M, Ban J, Kofler R, Walker B, Davis S, Meltzer P, Kovar H: A molecular function map of Ewing's sarcoma. PLOS ONE 2009, 4:e5415.

24. Tirode F, Laud-Duval K, Prieur A, Delorme B, Charbord P, Delattre O: Mesenchymal stem cell features of Ewing tumors. Cancer Cell 2007, 11:421-429.

25. Torchia EC, Jaishankar S, Baker SJ: Ewing tumor fusion proteins block the differentiation of pluripotent marrow stromal cells. Cancer Res 2003, 63:3464-3468

26. Riggi N, Cironi L, Provero P, Suva ML, Kaloulis K, Garcia-Echeverria C, Hoffmann F, Trumpp A, Stamenkovic I: Development of Ewing's sarcoma from primary bone marrow-derived mesenchymal progenitor cells. Cancer Res 2005, 65:11459-11468

27. Riggi N, Suva ML, Suva D, Cironi L, Provero P, Tercier S, Joseph JM, Stehle JC, Baumer K, Kindler V, Stamenkovic I: EWS-FLI-1 expression triggers a Ewing's sarcoma initiation program in primary human mesenchymal stem cells. Cancer Res 2008, 68:2176-2185.

28. Hancock JD, Lessnick SL: A transcriptional profiling meta-analysis reveals a core EWS-FLI gene expression signature. Cell Cycle 2007, 7:250-256.

29. Jawad MU, Cheung MC, Min ES, Schneiderbauer MM, Koniaris LG, Scully SP: Ewing sarcoma demonstrates racial disparities in incidence-related and sex-related differences in outcome: an analysis of 1631 cases from the SEER database, 1973-2005. Cancer 2009, 115:3526-3536.

30. Henderson SR, Guiliano D, Presneau N, McLean S, Frow R, Vujovic S, Anderson
J, Sebire N, Whelan J, Athanasou N, Flanagan AM, Boshoff C: A molecular map of mesenchymal tumors. Genome Biol 2005, 6:R76

31. Schaefer KL, Eisenacher M, Braun Y, Brachwitz K, Wai DH, Dirksen U, LanversKaminsky C, Juergens H, Herrero D, Stegmaier S, Koscielniak E, Eggert A, Nathrath M, Gosheger G, Schneider DT, Bury C, Diallo-Danebrock R, Ottaviano $L$, Gabbert HE, Poremba C: Microarray analysis of Ewing's sarcoma family of tumours reveals characteristic gene expression signatures associated with metastasis and resistance to chemotherapy. Eur J Cancer 2008, 44:699-709.

32. Scotlandi K, Remondini D, Castellani G, Manara MC, Nardi F, Cantiani L, Francesconi M, Mercuri M, Caccuri AM, Serra M, Knuutila S, Picci P: Overcoming resistance to conventional drugs in Ewing sarcoma and identification of molecular predictors of outcome. J Clin Oncol 2009, 27:2209-2216.

33. Knoop LL, Baker SJ: EWS/FLI alters 5'-splice site selection. J Bio/ Chem 2001, 276:22317-22322.

34. Sanchez G, Delattre O, Auboeuf D, Dutertre M: Coupled alteration of transcription and splicing by a single oncogene: boosting the effect on cyclin D1 activity. Cell Cycle 2008, 7:2299-2305.

35. Chansky HA, Hu M, Hickstein DD, Yang L: Oncogenic TLS/ERG and EWS/Fli-1 fusion proteins inhibit RNA splicing mediated by YB-1 protein. Cancer Res 2001, 61:3586-3590.

36. Deloulme JC, Prichard L, Delattre O, Storm DR: The prooncoprotein EWS binds calmodulin and is phosphorylated by protein kinase $\mathrm{C}$ through an IQ domain. J Bio/ Chem 1997, 272:27369-27377.

37. Knoop LL, Baker SJ: The splicing factor U1C represses EWS/FLI-mediated transactivation. J Biol Chem 2000, 275:24865-24871.

38. Watanabe G, Nishimori H, Irifune H, Sasaki Y, Ishida S, Zembutsu H, Tanaka T, Kawaguchi S, Wada T, Hata J, Kusakabe M, Yoshida K, Nakamura Y, Tokino T: Induction of tenascin-C by tumor-specific EWS-ETS fusion genes. Genes Chromosomes Cancer 2003, 36:224-232.

39. Fukuma M, Okita H, Hata J, Umezawa A: Upregulation of Id2, an oncogenic helix-loop-helix protein, is mediated by the chimeric EWS/ets protein in Ewing sarcoma. Oncogene 2003, 22:1-9.

40. Nakatani F, Tanaka K, Sakimura R, Matsumoto Y, Matsunobu T, Li X, Hanada M, Okada T, Iwamoto Y: Identification of p21WAF1/CIP1 as a direct target of EWS-Fli1 oncogenic fusion protein. J Biol Chem 2003, 278:15105-15115.

41. Prieur A, Tirode F, Cohen P, Delattre O: EWS/FLI-1 silencing and gene profiling of Ewing cells reveal downstream oncogenic pathways and a crucial role for repression of insulin-like growth factor binding protein 3. Mol Cell Biol 2004, 24:7275-7283.

42. Abaan OD, Levenson A, Khan O, Furth PA, Uren A, Toretsky JA: PTPL1 is a direct transcriptional target of EWS-FLI1 and modulates Ewing's sarcoma tumorigenesis. Oncogene 2005, 24:2715-2722.

43. Siligan C, Ban J, Bachmaier R, Spahn L, Kreppel M, Schaefer KL, Poremba C, Aryee DN, Kovar H: EWS-FLI1 target genes recovered from Ewing's sarcoma chromatin. Oncogene 2005, 24:2512-2524.

44. Wakahara K, Ohno T, Kimura M, Masuda T, Nozawa S, Dohjima T, Yamamoto T, Nagano A, Kawai G, Matsuhashi A, Saitoh M, Takigami I, Okano Y, Shimizu K: EWS-Fli1 up-regulates expression of the Aurora A and Aurora B kinases. Mol Cancer Res 2008, 6:1937-1945.

45. Beauchamp E, Bulut G, Abaan O, Chen K, Merchant A, Matsui W, Endo Y, Rubin JS, Toretsky J, Uren A: GLI1 is a direct transcriptional target of EWS-FLI1 oncoprotein. J Biol Chem 2009, 284:9074-9082.

46. Richter GH, Plehm S, Fasan A, Rossler S, Unland R, Bennani-Baiti IM, Hotfilder M, Lowel D, von Luettichau I, Mossbrugger I, Quintanilla-Martinez L, Kovar H, Staege MS, Muller-Tidow C, Burdach S: EZH2 is a mediator of EWS/FLI1 driven tumor growth and metastasis blocking endothelial and neuroectodermal differentiation. Proc Natl Acad Sci USA 2009, 106:5324-5329.

47. Guillon N, Tirode F, Boeva V, Zynovyev A, Barillot E, Delattre O: The oncogenic EWS-FLI1 protein binds in vivo GGAA microsatellite sequences with potential transcriptional activation function. PLOS ONE 2009, 4:e4932.

48. Gangwal K, Sankar S, Hollenhorst PC, Kinsey M, Haroldsen SC, Shah AA, Boucher KM, Watkins WS, Jorde LB, Graves BJ, Lessnick SL: Microsatellites as EWS/FLI response elements in Ewing's sarcoma. Proc Natl Acad Sci USA 2008, 105:10149-10154.

49. Luo W, Gangwal K, Sankar S, Boucher KM, Thomas D, Lessnick SL: GSTM4 is a microsatellite-containing EWS/FLI target involved in Ewing's sarcoma oncogenesis and therapeutic resistance. Oncogene 2009, 28:4126-4132.

50. Gangwal K, Lessnick SL: Microsatellites are EWS/FLI response elements: genomic "junk" is EWS/FLI's treasure. Cell Cycle 2008, 7:3127-3132.

51. Owen LA, Kowalewski AA, Lessnick SL: EWS/FLI mediates transcriptional 
repression via NKX2.2 during oncogenic transformation in Ewing's sarcoma. PLOS ONE 2008, 3:e1965.

52. Burdach S, Plehm S, Unland R, Dirksen U, Borkhardt A, Staege MS, MullerTidow C, Richter GH: Epigenetic maintenance of stemness and malignancy in peripheral neuroectodermal tumors by EZH2. Cell Cycle 2009, 8:1991-1996.

53. Lin PP, Pandey MK, Jin F, Xiong S, Deavers M, Parant JM, Lozano G: EWS-FLI1 induces developmental abnormalities and accelerates sarcoma formation in a transgenic mouse model. Cancer Res 2008, 68:8968-8975.

54. Torchia EC, Boyd K, Rehg JE, Qu C, Baker SJ: EWS/FLI-1 induces rapid onset of myeloid/erythroid leukemia in mice. Mol Cell Biol 2007, 27:7918-7934

55. Codrington R, Pannell R, Forster A, Drynan LF, Daser A, Lobato N, Metzler M, Rabbitts TH: The Ews-ERG fusion protein can initiate neoplasia from lineage-committed haematopoietic cells. PLoS Bio/ 2005, 3:e242.

56. Roberts P, Burchill SA, Brownhill S, Cullinane CJ, Johnston C, Griffiths MJ, McMullan DJ, Bown NP, Morris SP, Lewis IJ: Ploidy and karyotype complexity are powerful prognostic indicators in the Ewing's sarcoma family of tumors: a study by the United Kingdom Cancer Cytogenetics and the Children's Cancer and Leukaemia Group. Genes Chromosomes Cancer 2008 47:207-220.

57. Savola S, Klami A, Tripathi A, Niini T, Serra M, Picci P, Kaski S, Zambelli D, Scotlandi K, Knuutila S: Combined use of expression and CGH arrays pinpoints novel candidate genes in Ewing sarcoma family of tumors. BMC Cancer 2009, 9:17.

58. Hattinger CM, Potschger U, Tarkkanen M, Squire J, Zielenska M, KiuruKuhlefelt S, Kager L, Thorner P, Knuutila S, Niggli FK, Ambros PF, Gadner H, Betts DR: Prognostic impact of chromosomal aberrations in Ewing tumours. Br J Cancer 2002, 86:1763-1769.
59. Maurici D, Perez-Atayde A, Grier HE, Baldini N, Serra M, Fletcher JA: Frequency and implications of chromosome 8 and 12 gains in Ewing sarcoma. Cancer Genet Cytogenet 1998, 100:106-110.

60. Huang HY, Illei PB, Zhao Z, Mazumdar M, Huvos AG, Healey JH, Wexler LH, Gorlick R, Meyers P, Ladanyi M: Ewing sarcomas with p53 mutation or p16/p14ARF homozygous deletion: a highly lethal subset associated with poor chemoresponse. J Clin Oncol 2005, 23:548-558.

61. Klevernic IV, Morton S, Davis RJ, Cohen P: Phosphorylation of Ewing's sarcoma protein (EWS) and EWS-Fli1 in response to DNA damage. Biochem J 2009, 418:625-634.

62. Bachmaier R, Aryee DN, Jug G, Kauer M, Kreppel M, Lee KA, Kovar H: O-GlcNAcylation is involved in the transcriptional activity of EWS-FLI1 in Ewing's sarcoma. Oncogene 2009, 28:1280-1284.

63. Stegmaier K, Wong JS, Ross KN, Chow KT, Peck D, Wright RD, Lessnick SL, Kung AL, Golub TR: Signature-based small molecule screening identifies cytosine arabinoside as an EWS/FLI modulator in Ewing sarcoma. PLoS Med 2007, 4::122.

64. DuBois SG, Krailo MD, Lessnick SL, Smith R, Chen Z, Marina N, Grier HE, Stegmaier K: Phase II study of intermediate-dose cytarabine in patients with relapsed or refractory Ewing sarcoma: a report from the Children's Oncology Group. Pediatr Blood Cancer 2009, 52:324-327.

doi:10.1186/gm129

Cite this article as: Kovar H: Downstream EWS/FLI1 - upstream Ewing's sarcoma. Genome Medicine 2010, 2:8 